\title{
Relative Asymptotic Regularity and Fixed Points in Fuzzy 2-Metric Spaces
}

\author{
Mohit Kumar* \\ Department of Mathematics and Statistics, \\ Gurukula Kangri Vishwavidyalaya, \\ Haridwar (UK), India
}

\author{
Ritu Arora \\ Department of Mathematics and Statistics, \\ Gurukula Kangri Vishwavidyalaya, \\ Haridwar (UK), India
}

\begin{abstract}
In this paper, we established fixed point theorems for two and three self-maps of a complete fuzzy 2-metric space. The contractive definition is a generalization of Hardy-Rogers and the commuting condition of Jungck is replaced by the concept of weakly commuting. The notion of relative asymptotic regularity of a sequence in a fuzzy 2-metric space is introduced and fixed point theorems for two and three selfmappings of a complete fuzzy 2-metric space is proved. Further, a result for a pair of weakly commuting mappings and relative asymptotically regular sequence is presented in complete fuzzy 2-metric space.
\end{abstract}

\section{Keywords}

Fixed point, weakly commuting mapping, asymptotically regular mapping.

\section{AMS Subject Classification 54H25; 47H10; 47S40; 54A40}

\section{INTRODUCTION}

The notion of fuzzy set was introduced by Zadeh [15] in 1965 To use the concept in topology and analysis, many authors have extensively introduced the theory of fuzzy sets and applications. The fuzzy metric space was introduced by Kramosil and Michalek [10] in 1975. Grabiec [16] stated the contraction principle in fuzzy metric spaces in 1988 . Moreover, George and Veeramani [2] modified the notion of fuzzy metric spaces with the help of t-norms in 1994 . Gahler [19] introduced 2-metric spaces in a series of his papers. Sharma, Sharma and Ieski [12] investigated for the first time, contractive type mappings in 2-metric spaces. In 1982 Fisher [6] studied some related fixed point theorems on two metric spaces. Since many authors such as Aliouche and Fisher [1], R. K. Namdeo, S. Jain, B. Fisher [18], Telci [17] and others proved some related fixed point theorems in 2-metric and fuzzy metric spaces. S. Sharma [21] proved a common fixed point theorem for three mappings in fuzzy 2-metric spaces. We are following the notion of relative asymptotic regularity of a sequence in a metric space is introduced by RhoadesSessa-Khan-Khan [4] in 1984 .

In this paper, we prove fixed point theorems for three self maps in fuzzy 2-metric space and we use the asymptotic regularity of sequence in a fuzzy 2 -metric space.

\section{PRELIMINARIES}

2.1. Definition

(Schweizer and Sklar [22]) A binary operation $*:[0,1] \times[0,1] \rightarrow[0,1]$ is a continuous t-norm if $*$ satisfies the following conditions

[B.1] * is commutative and associative

[B.2] * is continuous

[B.3] $a^{*} 1=a \forall a \in[0,1]$

[B.4] $a^{*} b \leq c^{*} d$ whenever $a \leq c, b \leq d$ and $a, b, c, d \in[0,1]$.

\subsection{Definition}

(A. George and P. Veeramani [2] ) the $3-\operatorname{tuple}(X, M, *)$ is called a fuzzy metric space if $X$ is an arbitrary non -empty set, $*$ is a continuous t-norm and $M$ is a fuzzy metric in $X^{2} \times[0, \infty] \rightarrow[0,1]$, satisfying the following conditions: for all $x, y, z \in X$, and $t, s>0$.

[FM.1] $M(x, y, 0)=0$

[FM.2] $M(x, y, t)=1 \forall t>0$ if and only if $x=y$.

[FM.3] $M(x, y . t)=M(y, x, t)$

[FM.4] $M(x, y, t) * M(y, z, s) \leq M(x, z, t+s)$

[FM.5] $M(x, y, \square):[0, \infty] \rightarrow[0,1]$ is left continuous

[FM.6] $\lim _{n \rightarrow \infty} M(x, y, t)=1$.

2.3. Definition (A. George and P. Veeramani [2]) Let $\left(X, M,{ }^{*}\right)$ be a fuzzy metric space and let a sequence $\left\{x_{n}\right\}$ in $X$ is said to be converge to $x \in X$ if $\lim _{n \rightarrow \infty} M\left(x_{n}, x, t\right)=1$, for each $t>0$.

2.4. Definition (A. George and P. Veeramani [2] ) A sequence $\left\{x_{n}\right\}$ in $X$ is called Cauchy sequence if $\lim _{n \rightarrow \infty} M\left(x_{n}, x_{n+p}, t\right)=1$, for each $t>0$, and $p=1,2,3, \cdots$

2.5. Definition (A. George and P. Veeramani [2] ) A fuzzy metric space $(X, M, *)$ is said to be complete if every Cauchy sequence in $X$ is convergent in $X$. 
A fuzzy metric space in which every Cauchy sequence is convergent is called complete. It is called compact if every sequence contains a convergent subsequence.

2.6. Definition (A. George and P. Veeramani[2]) A self mapping $T: X \rightarrow X$ is called fuzzy contractive mapping if $M(T x, T y, t)>M(x, y, t)$ for each $x \neq y \in X$, and $t>0$.

2.7. Definition A binary operation

$*:[0,1] \times[0,1] \times[0,1] \rightarrow[0,1]$ is called a continuous t-norm if $([0,1], *)$ is an abelian topological monoid with unit 1 such that $a_{1} * b_{1} * c_{1} \leq a_{2} * b_{2} * c_{2}$ when $\quad a_{1} \leq a_{2}, b_{1} \leq b_{2}, c_{1} \leq c_{2}$ for $a_{1}, a_{2}, b_{1}, b_{2}$ and $c_{1}, c_{2}$ are in $[0,1]$.

2.8. Definition The 3-tuple $(X, M, *)$ is called a fuzzy 2metric space if $X$ is an arbitrary set, * is continuous t-norm and $M$ is a fuzzy set in $X^{3} \times[0, \infty)$ satisfying the following conditions; for all $x, y, z, u \in X$ and $t_{1}, t_{2}, t_{3}>0$

[FM-1] $M(x, y, z, 0)=0$

[FM-2] $M(x, y, z, t \neq 1 \neq$ and when at least two of the three points are equal

[FM-3] $M(x, y, z, t)=M(x, z, y, t)=M(y, z, x, t)$

(Symmetry about three variables)

$$
\text { [FM-4] } \begin{aligned}
M\left(x, y, z, t_{1}+t_{2}+t_{3}\right) \geq & M\left(x, y, u, t_{1}\right) * M\left(x, u, z, t_{2}\right) \\
& * M\left(u, y, z, t_{3}\right)
\end{aligned}
$$

(This corresponds to tetrahedron inequality in 2-metric space)

The function value $M(x, y, z, t)$ may be interpreted as the probability the area of triangle is less than 1 .

[FM-5] $M(x, y, z, \square:[0,1) \rightarrow[0,1]$ is left continuous.

2.9. Definition Let $(X, M, *)$ be a fuzzy 2 -metric space, then

(I) A sequence $\left\{x_{n}\right\}$ in fuzzy 2-metric space $X$ is said to be convergent to a point $x \in X$ if $\lim M\left(x_{n}, x, a, t\right)=1$ for all $a \in X$ and $t>0$.

(II) A sequence $\left\{x_{n}\right\}$ in fuzzy 2-metric space $X$ is called Cauchy sequence, if

$$
\lim _{n \rightarrow \infty} M\left(x_{n+p}, x_{n}, a, t\right)=1 \text { for all } a \in X \text { and } t>0, p>0 .
$$

(III) A fuzzy 2-metric space in which every Cauchy sequence is convergent is said to be complete.

2.10. Definition A function $M$ is continuous in fuzzy 2metric space if and only if whenever $x_{n} \rightarrow x, y_{n} \rightarrow y$ then $\lim _{n \rightarrow \infty} M\left(x_{n}, y_{n}, a, t\right)=M(x, y, a, t)$ for all $a \in X$ and $t>0$.

2.11. Definition Two mapping $P$ and $S$ in fuzzy 2-metric space $X$ are weakly commuting if and only if $M(P S u, S P u, a, t) \geq M(P u, S u, a, t)$ for all $u, a \in X$ and $t>0$.

2.11. Definition Let $(X, M, *)$ be a fuzzy 2 -metric space and $P$ and $S$ be self-maps of $X$. A sequence $\left\{x_{n}\right\}$ in $X$ is said to be asymptotically regular with respect to $\{P, S\}$ if $\lim _{n \rightarrow \infty} M\left(P x_{n}, S x_{n}, a, t\right)=1$ for all $a \in X$ and $t>0$.

\section{MAIN RESULT}

Theorem 3.1 Let $P, S$ be self mappings of a complete fuzzy 2-metric space $X$ satisfying

$$
\begin{aligned}
& \text { (i) } M(P x, P y, a, t) \geq a_{1} M\left(S x, P x, a, \frac{t}{5}\right)+a_{2} M\left(S y, P y, a, \frac{t}{5}\right) \\
& +a_{3} M\left(S x, P y, a, \frac{t}{5}\right)+a_{4} M\left(S y, P x, a, \frac{t}{5}\right)+a_{5} M\left(S x, S y, a, \frac{t}{5}\right)
\end{aligned}
$$

for all $x, y, a \in X$

where the $a_{i}=a_{i}(x, y) \geq 0 \quad(i=1, \cdots, 5)$

(ii) $\{P, S\}$ is a weekly commuting pair

(iii) There exists a sequence $\left\{x_{n}\right\}$ in $X$ which is asymptotically regular with respect to $\{P, S\}$

(iv) $S$ and $P$ are continuous

Then $P$ and $S$ have a unique common fixed point.

Theorem 3.2 Let $P, S, T$ be self mappings of a complete fuzzy 2-metric space $X$ satisfying

$$
\begin{array}{r}
\text { (3.1) } M(P x, P y, a, t) \geq a_{1} M\left(S x, P x, a, \frac{t}{4}\right)+a_{2} M\left(T x, P x, a, \frac{t}{4}\right) \\
+a_{3} M\left(S y, P y, a, \frac{t}{4}\right)+a_{4} M\left(T y, P y, a, \frac{t}{4}\right) \text { for all } x, y, a \in X \\
\text { where } a_{1}, a_{2}, a_{3}, a_{4} \text { are non-negative bounded numbers such }
\end{array}
$$
that $a_{2}+a_{3} \leq 1$ and $a_{3}+a_{4} \leq 1$.

(3.2) $\{P, S\}$ and $\{P, T\}$ are weakly commuting pairs .

(3.3) There exists a sequence $\left\{x_{n}\right\}$ in $X$ which is asymptotically regular with respect to $\{P, S\}$ and $\{P, T\}$ both.

(3.4) $S$ and $T$ are continuous

if $M$ is continuous then $P, S$ and $T$ have a unique common fixed point.

Proof Let $\left\{x_{n}\right\}$ satisfying (3.2), then from (3.1)

$$
\begin{aligned}
M\left(P x_{n}, P x_{m}, a, t\right) & \geq a_{1} M\left(S x_{n}, P x_{n}, a, \frac{t}{4}\right)+a_{2} M\left(T x_{n}, P x_{n}, a, \frac{t}{4}\right) \\
+ & a_{3} M\left(S x_{m}, P x_{m}, a, \frac{t}{4}\right)+a_{4} M\left(T x_{m}, P x_{m}, a, \frac{t}{4}\right)
\end{aligned}
$$

making $m, n \rightarrow \infty$

$$
\lim _{n \rightarrow \infty} M\left(P x_{n}, P x_{m}, a, t\right)=1
$$

and this is true for every $a \in X$. Hence $\left\{P x_{n}\right\}$ is Cauchy sequence and so convergent. Call the limit $z$. Also

$$
\begin{aligned}
M\left(Q x_{n}, z, a, t\right) & \geq M\left(Q x_{n}, P x_{n}, a, \frac{t}{3}\right)+M\left(P x_{n}, z, a, \frac{t}{3}\right) \\
& +M\left(Q x_{n}, P x_{n}, z, \frac{t}{3}\right) \rightarrow 1 \text { as } n \rightarrow \infty .
\end{aligned}
$$

So, $Q x_{n} \rightarrow z$. Similarly $T x_{n} \rightarrow z$.

The continuity of $S$ and $T$ implies 
$S P x_{n} \rightarrow S z, S^{2} x_{n} \rightarrow S z, S T x_{n} \rightarrow S z, T P x_{n} \rightarrow T z$,

$T^{2} x_{n} \rightarrow T z$ and $T S x_{n} \rightarrow T z$.

From (3.1)

(3.5) $M\left(R S x_{n}, P T x_{n}, a, t\right) \geq a_{1} M\left(S^{2} x_{n}, P S x_{n}, a, \frac{t}{4}\right)$

$+a_{2} M\left(T S x_{n}, P S x_{n}, a \frac{t}{4}\right)+a_{3} M\left(S T x_{n}, P T x_{n}, a, \frac{t}{4}\right)$

$+a_{4} M\left(T^{2} x_{n}, P T x_{n}, a, \frac{t}{4}\right)$

$M\left(P S x_{n}, S z, a, t\right) \geq M\left(P S x_{n}, S P x_{n}, a, \frac{t}{3}\right)+M\left(S P x_{n}, S z, a, \frac{t}{3}\right)$

$$
\begin{aligned}
& +M\left(P S x_{n}, S P x_{n}, S z, \frac{t}{3}\right) \\
\geq & M\left(S x_{n}, P x_{n}, a, \frac{t}{3}\right)+M\left(S P x_{n}, S z, a, \frac{t}{3}\right) \\
+ & M\left(S x_{n}, P x_{n}, S z, \frac{t}{3}\right) \rightarrow 1 \text { as } n \rightarrow \infty .
\end{aligned}
$$

So $P S x_{n} \rightarrow S z$. Similarly $P T x_{n} \rightarrow T z$. Further

$$
\begin{aligned}
& M\left(S T x_{n}, T S x_{n}, a, t\right) \geq M\left(S T x_{n}, P S x_{n}, a, \frac{t}{3}\right) \\
+ & M\left(S T x_{n}, T S x_{n}, P S x_{n}, \frac{t}{3}\right)+M\left(P S x_{n}, T S x_{n}, a, \frac{t}{3}\right)
\end{aligned}
$$

$$
\begin{aligned}
M\left(S T x_{n}, T S x_{n}, a, t\right) \geq M\left(S T x_{n}, P S x_{n}, a, \frac{t}{3}\right) \\
+M\left(S T x_{n}, T S x_{n}, P S x_{n}, \frac{t}{3}\right)+M\left(P S x_{n}, P T x_{n}, a, \frac{t}{9}\right) \\
+M\left(P T x_{n}, T S x_{n}, a, \frac{t}{9}\right)+M\left(P S x_{n}, T S x_{n}, P T x_{n}, \frac{t}{9}\right)
\end{aligned}
$$

Using (3.5) and making $n \rightarrow \infty$,(3.6) obtains

$$
\begin{aligned}
M(S z, T z, a, t) & \geq \frac{1}{3}+\frac{1}{3}+a_{2} M\left(T z, S z, a, \frac{t}{36}\right) \\
& +a_{3} M\left(S z, T z, a, \frac{t}{36}\right)+\frac{1}{9}+\frac{1}{9} \\
& \geq \frac{2}{3}+\frac{2}{9}+\left(a_{2}+a_{3}\right) M\left(T z, S z, a, \frac{t}{36}\right) \rightarrow 1
\end{aligned}
$$

therefore $S z=T z$.

From (3.1)

$$
\begin{aligned}
& M\left(P T x_{n}, P z, a, t\right) \geq a_{1} M\left(S T x_{n}, P T x_{n}, a, \frac{t}{4}\right) \\
& \quad+a_{2} M\left(T^{2} x_{n}, P T x_{n}, a, \frac{t}{4}\right)+a_{3} M\left(S z, P z, a, \frac{t}{4}\right) \\
& \quad+a_{4} M\left(T z, P z, a, \frac{t}{4}\right)
\end{aligned}
$$

Making $n \rightarrow \infty$

$M(T z, P z, a, t) \geq\left(a_{3}+a_{4}\right) M\left(T z, P z, a, \frac{t}{4}\right)$.

Yielding $P z=T z$.

Again from (3.1)

$$
\begin{aligned}
M(P P z, & P z, a, t) \geq a_{1} M\left(S P z, P P z, a, \frac{t}{4}\right) \\
& +a_{2} M\left(T P z, P P z, a, \frac{t}{4}\right)+a_{3} M\left(S z, P z, a, \frac{t}{4}\right) \\
& +a_{4} M\left(T z, P z, a, \frac{t}{4}\right) \\
= & a_{1} M\left(S P z, P S z, a, \frac{t}{4}\right)+a_{2} M\left(T P z, P t z, a, \frac{t}{4}\right) \\
\geq & a_{1} M\left(S z, P z, a, \frac{t}{4}\right)+a_{2} M\left(T z, P z, a, \frac{t}{4}\right) \rightarrow 1
\end{aligned}
$$

(Since $\{P, S\}$ and $\{P, T\}$ are weakly commuting pairs)

Hence $P P z=P z$ i.e. $P z$ is a fixed point of $P$. Set $P z=u$. Then $P S z=P P z=P z=u$ and

$$
\begin{aligned}
M(S u, u, a, t) & =M(S P z, u, a, t) \\
& \geq M\left(S P z, P S z, a, \frac{t}{3}\right)+M\left(S P z, P S z, u, \frac{t}{3}\right) \\
+ & M\left(P S z, u, a, \frac{t}{3}\right) \\
\geq & M\left(S z, P z, a, \frac{t}{3}\right)+M\left(P z, S z, a, \frac{t}{3}\right) \\
+ & M\left(u, u, a, \frac{t}{3}\right) \rightarrow 1
\end{aligned}
$$

So $S u=u$. Similarly $T u=u$. Thus $P u=S u=T u=u$.

To prove the uniqueness of $u$ as a common fixed point of $P, S$ and $T$. Let $v$ be another common fixed point of $P, S$ and $T$. Then

$$
\begin{aligned}
M(P u, P v, a, t) & \geq a_{1} M\left(S u, P u, a, \frac{t}{4}\right)+a_{2} M\left(T u, P u, a, \frac{t}{4}\right) \\
& +a_{3} M\left(S v, P v, a, \frac{t}{4}\right)+a_{4} M\left(T v, P v, a, \frac{t}{4}\right) \rightarrow 1
\end{aligned}
$$

Hence $u=v$.

\section{CONCLUSION}

In this paper, we have proved some fixed point theorems for three self maps in fuzzy 2-metric space and we have used the asymptotic regularity of sequence in a fuzzy 2 -metric space.

\section{ACKNOWLEDGEMENT}

This research work is supported by the University Grant Commission (UGC) New Delhi, India under Rajiv Gandhi National Fellowship (RGNF) to the first author.

\section{REFERENCES}

[1] A. Aliouche, B. Fisher, Fixed point theorem for mappings satisfying implicit relation on two complete metric spaces, Applied Mathematics and Mechanics, 27 (9)(2006), 1217-1222.

[2] A. George and P. Veeramani, On some results in fuzzy metric spaces, Fuzzy Sets and systems, 64,395-399(1994).

[3] Amin Ahmed, Deepak Singh, Mayank Sharma, Naval Singh, Results on fixed point theorems in two fuzzy metric spaces, fuzzy 2-metric spaces using rational inequality, International Mathematical Forum, 5, 2010, no. $39,1949-1973$. 
[4] B. E. Rhoades, S. Sessa, M. S. Khan and M. D. Khan, Some fixed point theorems for Hardy-Rogers type mappings, Internat. J. Math. \& Math. Sci. Vol. 7 No. 1 (1984) $75-87$.

[5] B. Fisher, Fixed point on two metric spaces, Glasnik Mat. 16(1981)333-337.

[6] B. Fisher, Related fixed point on two metric spaces, Mathematics Seminar notes, Vol. 10 (1982)17-26.

[7] G. E. Hardy and T. D. Rogers, A generalization of a fixed point theorem of Reich, Canad. Math. Bull. $16,201-206$ (1973)

[8] G. Jungck, Commuting mappings and fixed points, Amer. Math. Monthly 83, 261-263(1976).

[9] H. W. Engl, Weak convergence of asymptotically regular sequence for non expansive mapping and connections with certain cheishef-centers, Nonlinear Analysis TMA $1(5), 495-501(1977)$.

[10] I. Kramosil and J. Michalek, Fuzzy metric and statistical metric spaces, Ky-bernetica 11 (1975), 326-334.

[11] Jinkyu Han, A common fixed point theorem on fuzzy 2metric spaces, Journal of the Chungcheong Mathematical Society, Vol. 23, No. 4, december 2010.

[12] K. Iseki, P. L. Sharma, B. K. Sharma, Contractive type mapping on 2-metric space, Math. Japonica 21(1976), $67-70$.

[13] K. P. R. Rao, A. Aliouche, G. R. Babu, Related fixed point theorems in fuzzy metric spaces, The Journal of Nonlinear Sciences and its Application, 1(3), (2008),194-202.
[14] K. P. R. Rao, N. Shrivasa Rao, T. Ranga Rao, J. R. Prasad, Fixed and related fixed point theorems in sequentially compact fuzzy metric spaces, Int. Journal of Math. Analysis, Vol. 2,(2008), no. 28,1353-1359.

[15] L. A. Zadeh, Fuzzy sets, Inform and Control 89 (1965), 338-353.

[16] M. Grabiec, Fixed points in fuzzy metric spaces, Fuzzy Sets and System 27 (1988), 385-389.

[17] M. Telci, Fixed points on two complete and compact metric spaces, Applied Mathematics and Mechanics, 22(5)(2001), $564-568$

[18] R. K. Namdeo, S. Jain, B. Fisher, A Related fixed point theorem for two pairs of mapping on two complete metric spaces, Hacettepe Journal of Mathematics and Statistics, Vol. 32 (2003)07-11.

[19] S. Gahler, 2-metrische reume und ihre topologische structure, Math. Nauhr. 26(1963/64)115-148.

[20] S. N. Mishra, N. Mishra and S. L. Singh, Common fixed point of maps in fuzzy metric space, Internat. J. Math. \& Math. Sci. 17 (1994), 253-258

[21] S. Sharma, On fuzzy metric spaces, Southeast Asian Bull. Of Math. 26(2002), no. 1,133-145.

[22] Schweizer and Sklar, Statistical metric spaces, Pac. J. Math. Vol.(10) (1960) 385-389.

[23] Zaheer K. Ansari, Rajesh Shrivastava, Gunjan Ansari, Arun Garg, Some fixed point theorems in fuzzy 2-metric spaces and fuzzy 3-metric spaces, Int. J. contemp. Math Science, Vol. 6,2011,no. 46,2291-2301. 